\title{
Análisis de la capacidad explicativa de las pruebas de detección de talento en el rendimiento en competición de jóvenes triatletas
}

\author{
Explanatory capacity triathletes performance through \\ talent detection of Spanish federation
}

\section{Análise do poder explicativo dos testes de deteçao de talento no desempenho en competiçao de jovens triatletas}

\author{
Alba Cuba-Dorado ${ }^{1}$, Oscar García-García y Antonio Hernández-Mendo ${ }^{2}$ \\ ${ }^{1}$ Facultad de Ciencias de la Educación y del Deporte. Universidad de Vigo y ${ }^{2}$ Facultad de Psicología. Universidad de Málaga
}

\begin{abstract}
Resumen: El objetivo es establecer la relación entre las pruebas de detección de talentos de la federación española de triatlón (FETRI) y los resultados obtenidos en el campeonato de Espańa del mismo año. La muestra fue formada por 489 triatletas federados (342 hombres, 147 mujeres, 271 junior y 218 cadetes). Las variables independientes han sido las pruebas que conforman el proceso de detección de talentos de la FETRI: 100m de natación estilo libre, $1000 \mathrm{~m}$ de natación estilo libre, $400 \mathrm{~m}$ lisos, $1000 \mathrm{~m}$ lisos, y se ha utilizado como variable dependiente el puesto obtenido en el Campeonato de Espańa de triatlón. Se implementó un análisis de componentes de varianza $(\mathrm{p}<0,05)$, usando procedimientos de mínimos cuadrados (Varcomp) y de máxima verosimilitud (GLM) y un análisis de generalizabilidad. Los resultados indican que el modelo es significativo $(\mathrm{p}=0,0251)$ y que tres variables resultan significativas para explicar el puesto obtenido en el campeonato de Espańa de triatlón (natación de 100 y 1000 metros y carrera de 1000 metros), sin embargo ninguna de sus interacciones resulta significativa, así como tampoco la carrera de 400 metros. El modelo presenta un excelente nivel de fiabilidad $\left(\mathrm{e}^{2}=1\right)$ y de generalizabilidad $(\Phi=1)$. En conclusión, el resultado en el Campeonato de Espańa no encuentra una explicación satisfactoria a través de las pruebas de detección de talentos marcadas por la FETRI por lo que una posible solución sería mantener las pruebas que han mostrado una alta capacidad explicativa, y sustituir a aquellas otras que no tienen esa capacidad por la realización de otras más cercanas al modelo de rendimiento del triatlón.
\end{abstract}

Palabras clave: Selección, triatlón, toma de tiempos, predicción de rendimiento.

Abstract: The aim of this study is determine the relationship between the talent detection tests conducted by the Spanish Federation of Triathlon (FETRI) and the results in the Spanish Championship of the same year. The sample was composed by 489 federated triathletes (342 men, 147 women, 271 junior y 218 cadet). The independent variables were the four test included in the talent detection program conducted by the FETRI: 100 metres freestyle swimming, $1000 \mathrm{~m}$ freestyle swimming, $400 \mathrm{~m}$ running and $1000 \mathrm{~m}$ running and the dependent variable were the place finish in the Spanish Championship of Triathlon. An analysis of variance components $(\mathrm{p}<0.05)$, by least squares procedure (Varcomp) and maximum likelihood (GLM) was implemented, and an analysis of generalizability was performed. The results show a significant model $(\mathrm{p}=0.0251)$ and three variables are significant in explaining of the final position in the Spanish Championship of Triathlon (100 and 1000 metres swimming and 1000 metres running), yet none of their interactions is significant, well as the 400 metres running. The levels of reliability $(\mathrm{e} 2=1)$ and generalizability $(\Phi=1)$ of the model are excellent. In conclusion, the result in the Spanish Championship not find a satisfactory explanation through the talent detection tests conducted by the Spanish Federation a possible solution would be to keep the tests have shown a high explanatory power, and replace those that don't have that ability by performing other test closer to the model of triathlon performance.

Key words: Talent spotting, triathlon, timekeeping, performance prediction.

Resumo: $\mathrm{O}$ objetivo deste estudo é determinar a relação entre os testes de detecção de talentos realizado pela Federaçáo Espanhola de Triatlo (FETRI) e os resultados no campeonato de Espanha do mesmo ano. A amostra foi composta por 489 triatletas federados (342 homes, 147 mulleres, 271 junior y 218 cadetes). As variáveis independentes foram as provas incluídos no programa de detecção de talentos realizado pela FETRI: 100 metros nado livre, $1000 \mathrm{~m}$ nado livre, $400 \mathrm{~m}$ de corrida e $1000 \mathrm{~m}$ de corrida e a variável dependente foi a posição no Campeonato de Espanha de Triatlo. Foi implementada a análise de componentes de variância $(\mathrm{p}<0,05)$, utilizando procedimentos mínimos quadrados (Varcomp) e máxima verossimilhança (GLM) e uma análise de generalizaçáo. Os resultados indicam que o modelo é significativo $(p=0,0251)$ e que três variáveis são significativas na explicação da posição final no Campeonato de Espanha de Triatlo (100 e 1000 metros de nataçáo e 1000 metros de corrida), mas nenhuma das suas interaçōes é significativo, nem os 400 metros em execução. O modelo proporciona um excelente nível de confiabilidade $(\mathrm{e} 2=1)$ e generalizaçăo $(\Phi=$ 1). Em conclusáo, os resultados do Campeonato de Espanha não encontrar uma explicação satisfatória através dos testes de detecção de talentos realizado pela Federação Espanhola de uma possível solução seria a de manter os testes demonstraram alto poder explicativo, e substituir aqueles que náo tem essa capacidade através da realização de outro teste mais próximo do modelo de desempenho triatlo.

Palavras-chave: seleção, triatlo, tempo, previsão de desempenho. 


\section{Introducción}

El triatlón es un deporte de resistencia que combina, de forma secuencial y en orden establecido, las disciplinas de natación, ciclismo y carrera, donde el cronómetro no se detiene en ningún momento durante la competición. Las distancias oficiales de la International Triathlon Union (2011) son sprint $(750 \mathrm{~m}-20 \mathrm{~km}-5 \mathrm{~km})$, corta $(1,5 \mathrm{~km}-40 \mathrm{~km}-10 \mathrm{~km})$, media $(1,9 \mathrm{a}$ $3 \mathrm{~km}-80$ a $90 \mathrm{~km}-20$ a $21 \mathrm{~km})$ y larga $(3,8$ a $4 \mathrm{~km}-120$ a $180 \mathrm{~km}-$ 30 a $42,2 \mathrm{~km})$. El triatlón es una combinación de tres tipos diferentes de locomoción humana donde cada una de ellas requiere un rendimiento distinto, aunque el potencial rendimiento en cada uno de los segmentos está determinado en gran medida por la capacidad aeróbica, la duración concreta de cada segmento requerirá al deportista unas características específicas a la hora de obtener un buen resultado (Bentley et al., 2008; Kovárová, Juric y Kovár, 2012).

En concreto, la distancia corta de triatlón es sobre la que se desarrollan los JJOO y la mayor parte de las competiciones de las series mundiales, su duración es aproximadamente de dos horas de modo que la capacidad aeróbica, entendida como el esfuerzo de una determinada intensidad que puede ser mantenido de forma casi exclusiva por procesos metabólicos aeróbicos, es uno de los factores fundamentales para mantener un alto rendimiento (Chavaren, Dorado, López, 1996; Hausswirth y Brisswalter, 2008).

En este sentido, se ha informado de la importancia de la evaluación del $\mathrm{VO}_{2} \max$, ya sea de forma relativa al peso corporal o respecto al porcentaje desarrollado en el umbral anaeróbico, para conocer la capacidad aeróbica de los triatletas y utilizarlo como uno de los indicadores principales del rendimiento del triatleta (Bentley, Millet, Vleck, y McNaughton, 2002; Hue, 2000 y 2003; Kovarova y Kovar, 2012; Millet, Dreano y Bentley, 2003; Millet, Vleck y Bentley, 2009; Van Schuylenbergh, Vanden Eynde y Hespel , 2004; Suriano y Bishop, 2010). Sin embargo, no ha sido el único factor del rendimiento señalado, ya que las características antropométricas del deportista parece ser otro, aunque Ackland et al. (1997); Gianfelici et al. (2012) y Landers et al. (2000 y 2013) sugieren que el perfil idóneo del triatleta no está claramente definido, parece evidente que para alcanzar el alto rendimiento en triatlón es importante tener un bajo porcentaje graso. En esta línea a la hora de hablar de factores de rendimiento en triatlón no se deben de tener en cuenta solamente factores físicos del deportista, ya que, Bentley et al. (2002); Delextrat et al. (2003); Hausswirth et al. (1999 y 2001) y Millet y Bentley (2004), señalan que la estrategia adoptada en competición juega un papel fundamental en el resultado final.

Un talento deportivo es un deportista que presenta una aptitud caracterizada por unas condiciones y presupuestos tanto físicos como psíquicos que con mucha probabilidad lo llevaran a alcanzar el alto nivel en un determinado tipo de deporte (Gaber y Ruoff, 1979). Una de las competencias de las federaciones deportivas espańolas es impulsar a los deportistas con talento para que lleguen a alcanzar el alto nivel deportivo (CSD, 2010). La selección de talentos en el triatlón adquiere gran relevancia, ya que permite optimizar los recursos disponibles para la captación y la promoción de talentos con el fin de diseñar la mejor estrategia para lograr buenos resultados a nivel deportivo (Bottoni, Gianfelici, Tamburri y Faina, 2011). El apoyo y la formación adecuados son esenciales para que las personas con una aptitud hacia un tipo de actividad puedan alcanzar su potencial. Los procesos de identificación de talentos tienen que reconocer el carácter evolutivo del talento y por tanto combinar la identificación de talentos con su proceso de desarrollo y no considerar únicamente los niveles de rendimiento sino otros factores como por ejemplo, el compromiso del deportista (Abbot y Collins, 2004).

El estudio de la selección de talentos en triatlón no se ha abordado centrándose en el desempeño global en el triatlón sino en la predicción de resultados a corto plazo, atendiendo a factores de rendimiento diferentes: antropométricos, fisiológicos, morfológicos y psicológicos (Kovárová y Kovár, 2012). Sin embargo, a la hora de evaluar el potencial de un deportista Eriksson, Krampe y Tesch-Römer (1993) plantean que la influencia de lo innato en el rendimiento deportivo es mucho menos reseñable que otros factores. Es por ello que se debería tener en cuenta que para conseguir un modelo fiable de cara a la predicción del rendimiento no solo se han de evaluar los aspectos físicos sino que también deben ser evaluados la capacidad mental, la velocidad de desarrollo de habilidades, la utilización de los recursos endógenos y exógenos, y la tolerancia a la carga y al estrés (Bottoni et al., 2011).

Además, uno de los mayores problemas que presenta la predicción de rendimiento es conocer la influencia de cada uno de los segmentos en una competición de triatlón ya que, es difícil transferir los resultados de una prueba de competición a otra donde varía el tipo de circuito, su longitud, la altitud o el clima en la prueba (Kovárová et al., 2012).

Desde nuestro conocimiento no hay estudios en los que se validen las pruebas de detección de talentos desarrolladas por las federaciones de triatlón. Mediante la revisión de los programas de detección de talento que publican las federaciones nacionales de distintos países, incluidos algunos como Australia y Gran Bretańa con grandes resultados a nivel internacional, hemos encontrado que utilizan en sus programas de detección de talentos pruebas con distancias mayores en la carrera a las utilizadas por la FETRI, diferenciándolas en muchos casos según la edad del triatleta. Por ejemplo en Gran Bretaña se realiza $1 \mathrm{~km}$ de carrera a los 12 años y asciende hasta los $3 \mathrm{~km}$ a los 17 y 18 años. En las pruebas de natación parece que la mayor parte de los países optan por incluir una prueba intermedia a las utilizadas por la FETRI como es el $400 \mathrm{~m}$. 
En la actualidad no se ha abordado de manera precisa el estudio de la relación que las pruebas de detección de talentos que se realizan en la actualidad puedan tener con el rendimiento en los triatletas, cadetes o juniors, por lo que el objetivo de este trabajo es establecer la relación que puede existir entre las pruebas de detección de talentos de la federación española de triatlón y los resultados obtenidos en el Campeonato de Espańa de la modalidad. A priori, podría considerarse que debe haber una correlación positiva entre los resultados obtenidos en las pruebas de detección y el resultado obtenido en el Campeonato de Espańa.

\section{Método}

\section{Diseño de Investigación}

El diseño de este trabajo, siguiendo a Ato, López y Benavente (2013), responde a una investigación empírica que ha seguido una estrategia asociativa para un estudio explicativo, ya que se persigue explorar la relación existente entre las variables que conforman la toma de tiempos de la FETRI y los resultados obtenidos en el Campeonato de España de triatlón.

Como variables independientes han sido utilizadas las pruebas que conforman el proceso de detección de talentos de la Federación Española de Triatlón:

- Nat100: Tiempo en segundos de 100m de natación, estilo libre, realizados en la prueba de la Toma de Tiempos de la FETRI.

- Nat1000: Tiempo en segundos de $1000 \mathrm{~m}$ de natación, estilo libre, realizados en la prueba de la Toma de Tiempos de la FETRI.

- Carr400: Tiempo en segundos de 400m lisos de carrera realizados en la prueba de la Toma de Tiempos de la FETRI.

- Carr1000: Tiempo en segundos de $1000 \mathrm{~m}$ lisos de carrera realizados en la prueba de la Toma de Tiempos de la FETRI.

Como variable dependiente se ha utilizado el puesto, es decir, el resultado obtenido en el Campeonato de Espańa de triatlón cadete o junior, masculino o femenino (PCE).

\section{Participantes}

La población objeto del estudio la constituyeron 489 participantes, 342 hombres $(69,9 \%)$ y 147 mujeres $(30,1 \%)$, de los cuales $271(55,4 \%)$ pertenecían a la categorías junior (17 a 19 años el 31 de diciembre del ańo de la prueba) y 218 a la cadete (44,5\%) (15 y 16 ańos el 31 de diciembre del ańo de la prueba), todos ellos triatletas federados que durante los ańos
2009, 2010, 2011 y 2012 participaron tanto en el Campeonato de España de su categoría como en la toma de tiempos de la Federación Española de Triatlón. Para ser incluidos en el estudio los deportistas debían haber realizado las 4 pruebas de la toma de tiempos y finalizado el Campeonato de España durante el mismo año. El protocolo de investigación observó las directrices marcadas por la conferencia de Helsinki sobre Investigaciones Biomédicas en Humanos (18th Medical Assembly, 1964; revisada en 2008 en Seúl), y las marcadas por la Ley Orgánica 15/1999, de 13 de diciembre, de Protección de Datos de Carácter Personal, en su revisión del 06/03/2011.

\section{Procedimiento}

Se recogieron los datos relativos a los resultados de las tomas de tiempos organizados por la FETRI y la clasificación obtenida en el Campeonato de Espańa de triatlón de los años 2009, 2010, 2011 y 2012. Los resultados de los años 2010 a 2102 fueron obtenidos de la web de la FETRI (triatlón.org), en documento público, sin embargo para la obtención de las clasificaciones de 2009 hubo que recurrir a solicitarlas por escrito a la federación espańola de triatlón, ya que no están disponibles online. Los datos recogidos de la toma de tiempos y de la clasificación del campeonato de España fueron cruzados con el fin de identificar a los triatletas presentes en ambos eventos y poder ser incluidos en el estudio. Una vez hecha esa comprobación se obtuvo una muestra final de 489 triatletas.

\section{Análisis de datos}

Se obtuvieron los estadísticos descriptivos, media y desviación típica con un intervalo de confianza al 95\% (IC 95\%). Se implementó un análisis de componentes de varianza $(\mathrm{p}<0,05)$, usando procedimientos de mínimos cuadrados (Varcomp) y de máxima verosimilitud (GLM); y posteriormente se realizó una simulación manteniendo constantes la media y la desviación típica con el fin de comprobar si lo resultados se mantenían al aumentar la muestra. Con el fin de obtener los indicadores de fiabilidad (e2) y de generalizabilidad $(\Phi)$ de las variables significativas se realizó un análisis de Generalizabilidad. Fueron utilizados el paquete estadístico SAS System para Windows v. 9.1. el software SAGT v.1. para el desarrollo del análisis de generalizabilidad.

\section{Resultados}

A continuación, en la tabla 1, se pueden observar los tiempos medios que han obtenido los triatletas participantes en las pruebas de selección de talentos de la federación española. 
Tabla 1. Tiempos medios y desviaciones típicas (IC 95\%) en segundos de las variables de pruebas de selección.

\begin{tabular}{lcccc}
\hline Variables & Mínimo & máximo & Media & SD \\
\hline Nat100 & 55 & 95 & 66,8 & 6,8 \\
Nat1000 & 670 & 1200 & 844,0 & 96,7 \\
Carr400 & 53 & 92 & 66,5 & 7,8 \\
Carr1000 & 156 & 281 & 194,8 & 24,0 \\
\hline
\end{tabular}

El análisis de componentes de varianza sobre el modelo Puesto campeonato de Espańa $=\mathrm{N} 1 * \mathrm{~N} 2 * \mathrm{C} 1 * \mathrm{C} 2$, donde "N1" es la prueba de natación 100 metros, "N2" es la prueba de natación 1000 metros, "C1" es la carrera de 400 metros lisos, y "C2" es la carrera de 1000 metros lisos, muestra que el modelo es significativo $(\mathrm{p}=0.0251)$ al igual que 3 variables, explicando el $99 \%$ de la varianza y con un coeficiente de variación de 0,9881 (tablas 2 y 3). El procedimiento con Mínimos Cuadrados (Varcomp) y Máxima Verosimilitud (GLM), ha estimado que los valores de error son iguales con ambos procedimientos para cada una de las variables estudiadas. Este hallazgo permite considerar que la muestra es normal, lineal y homocedástica.

Tabla 2. Análisis de varianza modelo puesto Campeonato de España=Nat100*Nat1000*Carr400* Carr1000.

\begin{tabular}{lccccc}
\hline \multicolumn{4}{l}{ Variable dependiente: Puesto campeonato de España (PCE) } & & \\
\hline Fuente & DF & Suma de cuadrados & Cuadrado de la media & F & F-Valor Pr > \\
Modelo & 487 & 246003.9703 & 505,1416 & 1010,28 & 0,0251 \\
Error & 1 & 0,5000 & 0,5000 & & \\
Total corregido & 488 & 246004,4703 & & & \\
& R-cuadrado & Coef Var & Raíz MSE & PCE Media \\
& 0,999998 & 0,988127 & 0,707107 & 71,56033 \\
\hline
\end{tabular}

Tabla 3. Análisis de varianza modelo PCE $=$ Nat100*Nat1000*Carr400* Carr1000.

\begin{tabular}{|c|c|c|c|c|c|}
\hline Fuente & DF & Tipo III SS & Cuadrado de la media & F-Valor & $\operatorname{Pr}>F$ \\
\hline N1 & 1 & 364,500000 & 364,500000 & 729,00 & 0,0236 \\
\hline $\mathrm{N} 2$ & 15 & 4877,500000 & 325,166667 & 650,33 & 0,0308 \\
\hline $\mathrm{N} 1{ }^{*} \mathrm{~N} 2$ & 0 & 0,000000 & . & . & . \\
\hline $\mathrm{C} 1$ & 0 & 0,000000 & . & . & . \\
\hline $\mathrm{N} 1{ }^{*} \mathrm{C} 1$ & 0 & 0,000000 & . & . & . \\
\hline $\mathrm{N} 2 * \mathrm{C} 1$ & 0 & 0,000000 & . & . & . \\
\hline $\mathrm{N} 1{ }^{*} \mathrm{~N} 2^{*} \mathrm{C} 1$ & 0 & 0,000000 & . & . & . \\
\hline $\mathrm{C} 2$ & 4 & 3354,000000 & 838,500000 & 1677,00 & 0,0183 \\
\hline $\mathrm{N} 1{ }^{*} \mathrm{C} 2$ & 0 & 0,000000 & . & . & . \\
\hline $\mathrm{N} 2 * \mathrm{C} 2$ & 0 & 0,000000 & . & . & . \\
\hline $\mathrm{N} 1 * \mathrm{~N} 2{ }^{*} \mathrm{C} 2$ & 0 & 0,000000 & . & . & . \\
\hline $\mathrm{C} 1 * \mathrm{C} 2$ & 0 & 0,000000 & . & . & . \\
\hline $\mathrm{N} 1{ }^{*} \mathrm{C} 1{ }^{*} \mathrm{C} 2$ & 0 & 0,000000 & . & . & . \\
\hline $\mathrm{N} 2{ }^{*} \mathrm{C} 1{ }^{*} \mathrm{C} 2$ & 0 & 0,000000 & . & . & . \\
\hline $\mathrm{N} 1{ }^{*} \mathrm{~N} 2{ }^{*} \mathrm{C} 1{ }^{*} \mathrm{C} 2$ & 0 & 0,000000 & . & . & . \\
\hline
\end{tabular}

Como se puede observar en la tabla 3, tres variables resultan significativas para explicar el puesto obtenido en el campeonato de España de triatlón (natación de 100 y 1000 metros y carrera de 1000 metros), sin embargo ninguna de sus interacciones resulta significativa, así como tampoco resulta significativa la carrera de 400 metros.
En la tabla 4 se presenta el análisis de generalizabilidad para determinar la fiabilidad de la estructura de datos obtenida con los triatletas y su capacidad de generalización. El modelo presenta un excelente nivel de fiabilidad $\left(\mathrm{e}^{2}=1\right)$ y de generalizabilidad $(\Phi=1)$. 
Tabla 4. Fiabilidad $\left(\mathrm{e}^{2}\right)$ generalizabilidad $(\Phi)$ y porcentajes de varianzas asociadas

\begin{tabular}{lcccccccc}
\hline $\begin{array}{l}\text { Fuentes de } \\
\text { variación }\end{array}$ & Suma de cuadrados & Grado de libertad & Cuadrado medio & Aleatorio & Mixtos & Corregidos & \% Varianza & Error estándar \\
\hline N1 & 364,500 & 1 & 364,500 & 4,556 & 4,556 & 4,556 & 7,201 & 3,720 \\
N2 & 4877,500 & 15 & 325,167 & 32,517 & 32,517 & 32,517 & 51,389 & 11,153 \\
C2 & 3354,000 & 4 & 838,500 & 26,203 & 26,203 & 26,203 & 41,411 & 15,128 \\
\hline$e^{2}=1$ & $\Phi=1$ & & & & & &
\end{tabular}

Los porcentajes de varianza de cada variable señalan que la prueba de natación de 1000 metros, estilo libre, es la que más porcentaje de varianza explica con un $51,3 \%$, seguida de la carrera de 1000 metros lisos con un $41,4 \%$, situándose muy lejos de estas pruebas el porcentaje de varianza explicada por la prueba de natación de 100 metros (7,2\%). No se han encontrado diferencias significativas en el análisis de varianza de cada variable entre los triatletas cadetes y junior.

\section{Discusión}

Los principales resultados seńalan que la muestra objeto de estudio es normal, lineal y homocedástica, además los niveles de generalizabilidad y fiabilidad de la estructura de datos de los triatletas son altos. Las pruebas de natación de 1000 metros y la prueba de carrera de 1000 metros lisos han mostrado tener una relación significativa $(\mathrm{p}<0,05)$, y una alta capacidad explicativa del puesto alcanzado en el campeonato de Espańa, con un porcentaje de varianza explicada del 51,3\% y del 41 , $4 \%$ respectivamente.

La elevada capacidad explicativa de las pruebas de natación 1000 metros y carrera de 1000 metros lisos, contrasta con la baja capacidad explicativa de la prueba de natación 100 metros o incluso con la no inclusión en el modelo de la prueba de carrera de 400 metros lisos. Las pruebas de 100 metros natación, estilo libre, y de carrera de 400 metros lisos se caracterizan por ser pruebas de resistencia de corta duración, donde existe un claro predominio de la vía energética anaeróbica sobre la aeróbica (Capelli, Pendergast y Termin, 1998; Hill, 1999; Gastin, 2001; Spencer y Gastin, 2001; Duffield, Dawson y Goodman, 2005a;). Sin embargo la prueba de carrera 1000 metros se trata de un esfuerzo de resistencia de media duración, donde la utilización de las vías anaeróbicas y aeróbicas se mantiene en equilibrio (Lacour, Padilla-Magunacelaya, Barthélémy y Dormois, 1990; Spencer y Gastin, 2001; Duffield et al., 2005ab), y la prueba de 1000 metros de nado, estilo libre que ya se considera un esfuerzo de larga duración donde la vía aeróbica es la más utilizada (Capelli et al., 1998; Gastin, 2001).

Este hecho hace que la capacidad explicativa que tienen las pruebas que se realizan en el programa de detección de talentos para explicar el resultado del Campeonato de Espańa de Triatlón sea limitada. Esta limitación podría ser atribuida a la presencia de las pruebas de componente marcadamente anaeróbico, como es el caso de la prueba de 400 metros lisos, ya que Chavaren et al. (1996) y Kovárova et al. (2012) han señalado que la capacidad de rendimiento en el triatlón está determinado por la capacidad aeróbica y no por la capacidad anaeróbica. En este sentido, resulta bastante gráfico señalar que cuanto mejor marca han tenido en la prueba de 100 metros natación (N1) peor rendimiento se obtiene en el campeonato de Espańa, lo que unido a la no inclusión de la prueba de 400 metros lisos (C1) ilustra la afirmación de Chavaren et al. (1996) y Kovárova et al. (2012)

No obstante, el modelo resulta ser significativo, probablemente debido a la inclusión de pruebas con marcado acento aeróbico como es el caso de la prueba de natación 1000 metros, cuyo esfuerzo representa una duración media de $844,08 \pm 96,764$ s y que estaría en consonancia con el tipo de pruebas propuestas por Bottoni et al. (2011) y Kovárová y Kóvar (2012) para predecir el rendimiento futuro de los triatletas. Estos autores proponen pruebas de carrera, sobre una distancia de entre 2 y $5 \mathrm{~km}$, donde se desarrollan esfuerzos de resistencia de media-larga duración, con un alto predominio de la vía aeróbica sobre la anaeróbica (Lacour et al., 1990; Duffield et al., 2005ab), y de natación, sobre una distancia de entre $200 \mathrm{~m}$ y $800 \mathrm{~m}$ donde se emplearían las mismas vías metabólicas (Capelli et al., 1998; Gastin, 2001; Laffite et al., 2004).

Se podrían sugerir otras causas por las que la capacidad explicativa que tienen estas pruebas es limitada como podría ser el no haber atendido a los distintos factores de rendimiento: antropométricos, fisiológicos, morfológicos y psicológicos, como indicaban Kovárová y Kóvar (2012) e incluso aspectos no innatos como la capacidad mental, la velocidad de desarrollo de habilidades, la utilización de los recursos endógenos y exógenos o la tolerancia a la carga y al estrés que señalaban Eriksson et al. (1993) y Bottoni et al. (2011).

Además, hay algunas cuestiones que tienen que ver con el formato de realización de las pruebas en condiciones diferentes a las de competición. En concreto, en el caso de las pruebas de natación se desarrollan en piscina con un individuo por calle, lo que difiere de forma notable de la natación en aguas abiertas, o en las pruebas de carrera la realización de ellas sin fatiga previa dista bastante del modelo de competición. Además, otras circunstancias propias de la competición, como 
pueden ser las transiciones o la táctica desarrollada, plantean la necesidad de incluir en alguna de las pruebas estas situaciones de competición, como se ha venido realizando en otros trabajos (Bentley, Millet, Vleck, y McNaughton, 2002; Hue, 2003; Millet, Dreano y Bentley, 2003; Van Schuylenbergh, Vanden Eynde y Hespel, 2004; Suriano y Bishop, 2010) y que podrían proporcionar una mayor capacidad explicativa del resultado alcanzado en competición.

Por otro lado, también se ha sugerido que el factor antropométrico (Landers et al., 2000; Ackland et al., 2007; Landers et al., 2013) y el psicológico (Kovárová y Kóvar, 2012) pueden condicionar el rendimiento del triatleta por lo que parece oportuno que algunas pruebas antropométricas y psicológicas podrían formar parte de la batería de pruebas que componen el programa de detección de talentos, como por ejemplo el porcentaje de grasa.

Otra cuestión pendiente sería la importancia que se le debe dar a cada prueba, es decir, si sería necesario ponderar el resultado en cada una de ellas debido por ejemplo a que el segmento de la carrera es mucho más influyente en el resultado final del triatlón como han señalado Fröhlich et al. (2008).

Con los resultados obtenidos se podría sugerir que sería útil diseñar una mejor estrategia para la detección de talentos, como proponían Bottoni et al. (2011) con el fin de identificar de mejor manera y sacar el máximo partido a los talentos deportivos. Esta estrategia podría estar encaminada a diseñar una batería que incluyan no solo pruebas de marcado acento bioenergético, sino también antropométricas o psicológicas, así como indicadores más cualitativos relacionados con la eficiencia de la brazada o de la zancada de carrera.

A pesar de las limitaciones de este estudio, como carecer de un desarrollo longitudinal, se podría decir que la influencia de las pruebas establecidas por la Federación Española de Triatlón a partir del programa potenciado desde el Consejo Superior de Deportes son limitadas y quizás se acercan poco a la realidad competitiva del triatlón, por lo que sería oportuno proponer una batería de pruebas que incluya algunas que se asemejen más a la realidad de la competición en el triatlón y prescindir de las que no han mostrado capacidad explicativa.

\section{Conclusión}

El resultado en el Campeonato de España no encuentra una explicación satisfactoria a través de las pruebas de detección de talentos marcadas por la Federación Española de Triatlón por lo que una posible solución sería mantener las pruebas que han mostrado una alta capacidad explicativa, y sustituir a aquellas otras que no tienen esa capacidad por la realización de otras pruebas más cercanas al modelo de rendimiento que presentan los triatletas.

\section{Aplicaciones prácticas}

Los resultados obtenidos sugieren investigar acerca de un nuevo modelo para el programa de detección de talentos con el ánimo de dar mayor validez al mismo. Será necesario identificar otras pruebas que permitan un alto grado de asociación entre el resultado obtenido con el rendimiento alcanzado en competición. Las pruebas actuales parece que no permiten detectar y seleccionar de forma fundamentada y satisfactoria talentos en triatlón como se ha venido sugiriendo desde diferentes foros de entrenadores y técnicos.

\section{Referencias}

1. Abbot, A. y Collins, D. (2004). Eliminating the dichotomy between theory and practice in talent identification and development: considering the role of psychology. Journal of Sport Sciences, 22 (5), 395-408.

2. Ackland, T.R., Blanksby, B. A., Landers, G. y Smith, D. (1997). Anthropometric profiles of Elite Triathletes. Journal of Science Medicine in Sport, 1, 51-56.

3. Ato, M., López, J.J. y Benavente, A. (2013). Un sistema de clasificación de los diseños de investigación en psicología. Anales de psicología, 29 (3), 1038-1059.

4. Bentley, D. J., Cox, G. R., Green, D. y Laursen, P. B. (2008). Maximising performance in triathlon: Applied physiological and nutritional aspects of elite and non-elite competitions. Journal of Science and Medicine in Sport, 11(4), 407-416.

5. Bentley, D. J., Millet, G. P., Vleck, V. E. y McNaughton, L. R. (2002). Specific aspects of contemporary triathlon implications for physiological analysis and performance. Sports Medicine, 32 (6), 345-359.

6. Bottoni, A., Gianfelici, A., Tamburri, R. y Faina, M. (2011). Talent selection criteria for olympic distance triathlon. Journal of Human Sport \& Exercise 6 (2), 293-304.

7. Capelli, C., Pendergast, D.R. y Termin, B. (1998). Energetics of swimming at maximal speeds in humans. European Journal of Applied Physiology, 78, 385-393.
8. Chavaren Cabrero, J., Dorado García, C. y López Calbet, J.A. (1996). Triatlón: factores condicionantes del rendimiento y del entrenamiento. Revista de Entrenamiento Deportivo. 10 (2), 29-37.

9. Consejo Superior de Deportes (2010). Historia tecnificación depor tiva. Retrieved 20/06/2013 from: http://www.csd.gob.es/csd/com peticion/03tecnificacionDeportiva/01Int/02EvHis/01his/?searchte $\mathrm{rm}=1989 \% 20 \mathrm{se} \% 20 \mathrm{crea}$

10. Delextrat, A., Tricot, V., Bernard, T., Vercruyssen, F., Hausswirth, C., y Brisswalter, J. (2003). Drafting during swimming improves efficiency during subsequent cycling. Medicine and Science in Sports and Exercise, 35(9), 1612-1619.

11. Duffield, R., Dawson, B. y Goodman, C. (2005a) Energy system contribution to 400-metre and 800-metre track running. Journal of Sports Sciences, 23(3), 299-307.

12. Duffield, R., Dawson, B. y Goodman, C. (2005b) Energy system con tribution to 1500- and 3000-metre track running. Journal of Sports Sciences, 23(10), 993-1002.

13. Eriksson, K., Krampe, R. y Tesch-Römer, C. (1993). The role of deliberate practice in the adquisition of expert performance. Phycological Review, 100(3), 363-406.

14. Gastin, P.B. (2001). Energy system interaction and relative contribution during maximal exercise. Sports Medicine, 31(10), 725-41. 
15. Hill, D.W. (1999). Energy system contributions in middle-distance running events. Journal of Sports Sciences, 17(6), 477-483.

16. Hue, O., Le Gallais, D., Chollet, D., y Prefaut, C. (2000). Ventilatory threshold and maximal oxygen uptake in present triathletes. $\mathrm{Ca}$ nadian Journal of Applied Physiology-Revue Canadienne De Physiologie Appliquee, 25(2), 102-113.

17. Hue, O. (2003). Prediction of drafted-triathlon race time from submaximal laboratory testing in elite triathletes. Canadian Journal of Applied Physiology, 28 (4), 547-560.

18. Fröhlich, M., Klein, K., Pieter, A., Emrich, E. y Gießing, J. (2008). Consequences of the Three Disciplines on the Overall Result in Olympic-distance Triathlon. International Journal of Sports Science and Engineering, 2(4), 204-210.

19. Gaber, H. y Ruoff, B. A. (1979) Zum problem Talentbestimmung im sport. Sportwissenschaft. 9, 164-180.

20. Gianfelici, A., Tamburri, R., Miglio, M., Bottoni, A., Bomprezzi, A., y Faina, M. (2012). Anthropometric and physiological profile of young male athletes of olympic triathlon. Medicina Dello Sport, 65(2), 167-172.

21. Hausswirth, C., y Brisswalter, J. (2008). Strategies for improving performance in long duration events: Olympic distance triathlon. Sports Medicine, 38(11), 881-891.

22. Hausswirth, C., Lehenaff, D., Dreano, P. y Savonen, K. (1999). Effects of cycling alone or in a sheltered position on subsequent running performance during a triathlon. Medicine and Science in Sports and Exercise, 31(4), 599-604.

23. Hausswirth, C., Vallier, J. M., Lehenaff, D., Brisswalter, J., Smith, D., Millet, G. y Dreano, P. (2001). Effect of two drafting modalities in cycling on running performance. Medicine and Science in Sports and Exercise, 33(3), 485-492.

24. Kovárová, L. y Kovár, K. (2012). Verification of the model of predisposition in triathlon -structural model of confirmative factor analysis. Acta Universitatis Palackianae Olomucensis, Gymnica 42 (3), 27-38.
25. Lacour J. R., Padilla-Magunacelaya S., Barthélémy J. C. y Dormois, D. (1990). The energetics of middle-distance running. European Journal of Applied Physiology and Occupational Physiology, 60(1), 38-43.

26. Laffite, L.P., Vilas-Boas, J.P., Demarle. A., Silva. J., Fernandes. R. y Billat. V.L. (2004). Changes in physiological and stroke parameters during a maximal $400-\mathrm{m}$ free-swimming test in elite swimmers. $\mathrm{Ca}$ nadian Journal of Applied Physiology 29(Suppl.), SI7-S31.

27. Landers, g., Blanksby, B., Ackland, T. y Smith, D. (2000). Morphology and performance of world championship triathletes. Anatomy $\mathrm{Hu}$ man and Biology. 27, 387-400.

28. Landers, G. J., Ong, K. B., Ackland, T. R., Blanksby, B. A., Main, L. C., y Smith, D. (2013). Kinanthropometric differences between 1997 world championship junior elite and 2011 national junior elite triathletes. Journal of Science and Medicine in Sport, 16(5), 444-449.

29. Millet, G. P. y Bentley, D. J. (2004). The physiological responses to running after cycling in elite junior and senior triathletes. International Journal of Sports Medicine, 25(3), 191-197.

30. Millet, G. P., Dreano, P. y Bentley, D. J. (2003). Physiological characteristics of elite short and long distance triathletes. European Journal of Applied Physiology, 88, 427-430.

31. Millet, G. P., Vleck, V. E. y Bentley, D. J. (2009). Physiological differences between cycling and running lessons from triathletes. Sports Medicine, 39(3), 179-206

32. Spencer M.R. y Gastin P.B. (2001). Energy system contribution during 200 to $1500 \mathrm{~m}$ running in highly trained athletes. Medicine and Science in Sports and Exercise, 33(1), 157-162

33. Suriano, R. y Bishop, D. (2010). Physiological attributes of triathletes. Journal of Science and Medicine in Sport, 13 (3), 340-347.

34. Van Schuylenbergh, R., Vanden Eynde, B. y Hespel, P. (2004). Prediction of sprint triathlon performance from laboratory tests. European Journal of Applied Physiology, 91, 94-99. 